\title{
Anti-dyslipidemic and antioxidant activities of different fractions of Pongamia pinnata (lin.) fruits
}

\author{
Gitika Bhatia - Anju Puri · Rakesh Maurya • \\ Prem PrakashYadav • Mohammad Mubin Khan • \\ Ashok Kumar Khanna · Jitender Kumar Saxena
}

Published online: 5 June 2008

(C) Birkhäuser Boston 2008

\section{Erratum to: Med Chem Res DOI 10.1007/s00044-007-9063-2}

Due to an author error, this paper published online with incorrect data in Table 3 and the Results section. The Results section appears below. See especially the last two sentences. The corrected Table 3 follows.

\section{Results}

Effect of $P$. pinnata fractions on hyperlipidemia

Administration of triton WR-1339 in rats induced marked hyperlipidemia, which was observed by a marked increase in plasma levels of Tc (3.0 fold), Tg ( 2.59 fold), and PL (3.56 fold) as compared to control (Table 1). Treatment of hyperlipidemic rats with P. pinnata fruits fractions A, B, C, and D at a dose of $250 \mathrm{mg} / \mathrm{kg}$ reversed the plasma levels of lipid. The lipid-lowering effect caused by fraction $\mathrm{C}$ was greater than that caused by the others as there was a decrease in plasma levels of Tc, Tg, and PL by $29 \%$, $21 \%$, and $21 \%$, respectively. Guggulipid at the same dose caused a lowering of plasma $\mathrm{Tc}, \mathrm{Tg}$, and PL by $34 \%, 36 \%$, and $38 \%$, respectively, and was therefore more active than fruits fractions. In other experiments dyslipidemia in hamster was produced by feeding with an HFD. The plasma levels of Tg, Tc, HDL-chol, glucose, and glycerol of

The online version of the original article can be found under doi:10.1007/s00044-007-9063-2.

G. Bhatia $(\bowtie) \cdot$ A. Puri · M. M. Khan · A. K. Khanna · J. K. Saxena

Division of Biochemistry, Central Drug Research Institute, Lucknow 226001, India

e-mail: gitikabhatia@yahoo.co.in

R. Maurya · P. PrakashYadav

Medicinal \& Process Development, C.D.R.I, Lucknow 226001, India 


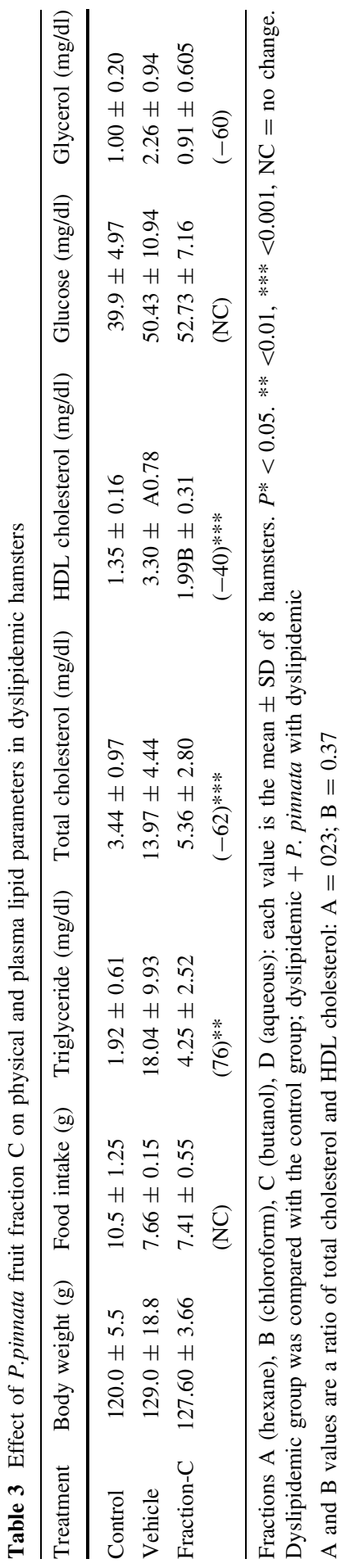


these animals were found to be elevated by $840 \%, 306 \%, 144 \%, 26 \%$, and $126 \%$ respectively as compared to the control. Administration of fruit fraction $\mathrm{C}$ at a dose of (500 mg/kg p.o.) simultaneously with HFD in hamster caused a decrease in the plasma levels of $\mathrm{Tg}(76 \%)$, Tc (62\%), and glycerol (60\%) respectively as compared to the dyslipidemic group (Table 3). 